\title{
Higgs boson to charm quark decay in vector boson fusion plus a photon
}

\author{
Ben Carlson๑, ${ }^{*}$ Tao Han, ${ }^{\dagger}$ and Sze Ching Iris Leung ${ }^{\ddagger}$ \\ PITT PACC, Department of Physics and Astronomy, University of Pittsburgh, \\ Pittsburgh, Pennsylvania 15260, USA
}

(Received 9 July 2021; accepted 23 September 2021; published 26 October 2021)

\begin{abstract}
Experimentally probing the charm-Yukawa coupling in the LHC experiments is important, but very challenging due to an enormous QCD background. We study a new channel that can be used to search for the Higgs decay $H \rightarrow c \bar{c}$, using the vector boson fusion (VBF) mechanism with an associated photon. In addition to suppressing the QCD background, the photon gives an effective trigger handle. We discuss the trigger implications of this final state that can be utilized in ATLAS and CMS. We propose a novel search strategy for $H \rightarrow c \bar{c}$ in association with VBF jets and a photon, where we find a projected sensitivity of about 13 times the SM charm-Yukawa coupling at $95 \% \mathrm{CL}_{s}$ at High Luminosity LHC (HL-LHC). Our result is comparable and complementary to existing projections at the HL-LHC. We also discuss the implications of increasing the center of mass collision energy to $30 \mathrm{TeV}$ and $100 \mathrm{TeV}$.
\end{abstract}

DOI: 10.1103/PhysRevD.104.073006

\section{INTRODUCTION}

Since the discovery of the Higgs boson $(H)$ by the ATLAS [1] and CMS [2] collaborations, determining its properties has become a high priority for the experiments at the LHC. Higgs boson couplings to weak gauge bosons are governed by the spontaneous symmetry breaking of the gauge theory, and have been well measured. However, the mass generation of fermions is a distinctive question. In the Standard Model (SM), fermion mass terms emerge from Higgs boson Yukawa interactions and thus the Yukawa couplings are proportional to the fermion mass. Therefore, it is crucial to establish the pattern of the Yukawa couplings to fermions in order to verify the SM and seek hints of Beyond-the-Standard-Model (BSM) physics. To date, Higgs couplings to third generation fermions have been observed to $b \bar{b}[3,4], t \bar{t}[5,6]$ and to $\tau^{+} \tau^{-}$[7-9]. Direct observations of the Higgs couplings to the second generation of fermions are thus of critical importance to further confirm the non-universal pattern of Yukawa couplings $[10,11]$. Because of its distinctive experimental signature, the decay mode $H \rightarrow \mu^{+} \mu^{-}$via the $g g$ fusion production [12] and the vector-boson fusion production (VBF) [13] is promising to be observed in the near future $[14,15]$. Testing

\footnotetext{
*bcarlson@pitt.edu

than@pitt.edu

SZL13@pitt.edu
}

Published by the American Physical Society under the terms of the Creative Commons Attribution 4.0 International license. Further distribution of this work must maintain attribution to the author(s) and the published article's title, journal citation, and DOI. Funded by SCOAP ${ }^{3}$. the Higgs Yukawa coupling to the charm quark $\left(y_{c}\right)$, on the other hand, is known to be challenging at hadron colliders due to the formidable QCD backgrounds.

At the LHC, billions of $p p$ collisions happen every second but only a small fraction of these events will be recorded due to limitations in data storage capacity and rate limitations of the detector readout electronics. The judicious selection of events as using triggers such as isolated leptons, photons, or jets with high transverse momentum, are needed to record events of physical interest. Searching for the decay mode $H \rightarrow c \bar{c}$ with limited energy for the decay products requires incorporating the Higgs boson production mechanism to develop an efficient trigger strategy. There are currently two experimental probes of the charm-Yukawa coupling at the LHC. One approach is to use Higgs association production with a leptonically decaying $Z$ boson ( $Z H$ channel) $[16,17]$. The $Z H$ channel provides a bound on $\mu$ of 110 for $36.1 \mathrm{fb}^{-1}$ of data, where $\mu$ is defined as the ratio of the new physics cross section and the SM expectation. An extrapolation of the ATLAS analysis leads to a projection of $\mu<6$ using $3 \mathrm{ab}^{-1}$ at the HL-LHC [18]. A recent preliminary result from ATLAS improves the sensitivity to $\mu<26$ by utilizing $139 \mathrm{fb}^{-1}$ and the leptonic decays of the $W$ boson as well as $Z$ to invisible decays [19]. A similar result from CMS also incorporating associated production with a $W$ boson, $Z$ to invisible decay modes and utilizing substructure techniques yields an observed constraint of $\mu<70$ [20]. The LHCb experiment has provided limits using $1.98 \mathrm{fb}^{-1}$ of data, providing an observed constraint of $\mu<6400$ [21], with a projection of an upper limit on the $\mu$ of 50 after collecting $300 \mathrm{fb}^{-1}$ of data at $14 \mathrm{TeV}$ assuming no improvements in the detector performance or analysis [22]. 
TABLE I. Summary of existing search results at the LHC (upper two rows) and the HL-LHC projection (lower two rows). The CMS entry marked with ${ }^{*}$ is scaled from the reported $\mu$ value to higher luminosity. The entries marked with ${ }^{\dagger}$ were computed from the reported $\mu$ values (see Sec. III C.) The entry marked with ${ }^{\dagger \dagger}$ is scaled according to the description in the text following [25].

\begin{tabular}{lcccc}
\hline \hline Channel & \multicolumn{3}{c}{$\sigma(V H) \times \mathcal{B}(H \rightarrow c \bar{c})$} & $\mathcal{B}(H \rightarrow J / \psi \gamma)$ \\
\hline Experiment & ATLAS & $\mathrm{CMS}$ & $\mathrm{LHCb}$ & ATLAS \\
\hline Data set & $13 \mathrm{TeV}$ & $13 \mathrm{TeV}$ & $8 \mathrm{TeV}$ & $13 \mathrm{TeV}$ \\
& $139 \mathrm{fb}^{-1}$ & $35.9 \mathrm{fb}^{-1}$ & $1.98 \mathrm{fb}^{-1}$ & $36.1 \mathrm{fb}^{-1}$ \\
$\mu\left(95 \% \mathrm{CL}_{s}\right)$ & $26[19]$ & $70[20]$ & $6400[21]$ & $120[29]$ \\
HL-LHC on $\mu$ & $6.3[18]$ & $7.7^{*}$ & $50\left(300 \mathrm{fb}^{-1}\right)[33]$ & $15[31]$ \\
HL-LHC on $\kappa_{c}$ & $2.7^{\dagger}$ & $3.1^{\dagger}$ & $7\left(300 \mathrm{fb}^{-1}\right)[22]$ & $50^{\dagger \dagger}$ \\
\hline \hline
\end{tabular}

Another approach does not rely on tagging charm quarks from the Higgs decay, but instead uses the decay of a Higgs boson $H \rightarrow J / \psi+\gamma$ [23-27], a process that has been searched for by ATLAS [28,29] and CMS [30]. This process gives a looser bound on charm-Yukawa coupling of 50 times the SM prediction even at the HL-LHC [31], due to the contamination from $H \rightarrow \gamma \gamma^{*}$ with a vector meson dominance in $\gamma^{*}-J / \psi$ mixing, which is about an order of magnitude larger than that from the direct $H c \bar{c}$ coupling $[23,25,32]$.

In Table I, we collect the current results from the LHC searches (upper two rows) and the HL-LHC projection (lower two rows). For the $V H$ channel, the HL-LHC projections at CMS are estimated by simply scaling for the signal strength $\mu$ from the increase of the luminosity. We further translate the results to estimate the sensitivity to the modification from the SM coupling $\kappa_{c}=y_{c}^{\mathrm{BSM}} / y_{c}^{\mathrm{SM}}$ as described in Sec. III C. For the channel $H \rightarrow J / \psi+\gamma, \kappa_{c}$ does not have a simple relation with $\mu$ due to the contamination from $H \rightarrow \gamma \gamma^{*}$ as noted above. It is instead estimated using Eq. (53a) in [25].

Rather than search for $H \rightarrow c \bar{c}$, it has also been proposed to constrain $y_{c}$ by requiring a charm tag in the production $g c \rightarrow H c$ with $H \rightarrow \gamma \gamma$. This channel yields a $95 \% \mathrm{CL}_{s}$ limit on $\kappa_{c}$ ranging from 2.6 to 3.9 at HL-LHC, depending on theoretical uncertainties [34]. An attempt to perform a global fit for the Higgs couplings may lead to a tighter bound on the charm-Yukawa coupling [27,35-37], with a few modeldependent assumptions. In particular, a $95 \% \mathrm{CL}_{s}$ upper bound on $\kappa_{c}$ of 1.2 at HL-LHC is claimed in [36], obtained from the upper limit of branching ratio of Higgs boson decays to untagged BSM particles assuming $\left|\kappa_{V}\right| \leq 1$. Exploiting kinematic information of the Higgs boson, such as transverse momentum distribution and rapidity distribution, was proposed in probing the light-quark Yukawa couplings [38-40] and implemented in a combined fit by CMS [41]. The asymmetry between $W^{+}$and $W^{-}$production has also been proposed to constrain $y_{c}[35,42]$. It has also been proposed to constrain $y_{c}$ using Di-Higgs production $[43,44]$. There are proposals to further enhance the sensitivity of $H \rightarrow c \bar{c}$ by utilizing an additional photon radiation [45-47].
In this work, we propose a novel approach to probe the Yukawa coupling of charm quark via VBF for the Higgs boson production with an additional photon. This work builds off of the idea of introducing a new subset of the VBF production mode utilizing photon radiation as an additional handle [48-54] for triggering and background suppression. The new channel we propose can provide complementary information to the existing searches using the $W H$ and $Z H$ channels.

The rest of the paper is organized as follows. We first lay out our search strategy in Sec. II, in particular a proposal for triggering the signal events. We then present our analyses and the results in Sec. III, including HL-LHC. We finally summarize our results and draw the conclusion in Sec. IV.

\section{PROPOSED SEARCH STRATEGY}

The decay branching fraction for $H \rightarrow c \bar{c}$ is about $3 \%$, which leads to a cross section about $0.1 \mathrm{pb}(1 \mathrm{pb})$ from the $\operatorname{VBF}(g g)$ production at the $13 \mathrm{TeV}$ LHC [55]. This yields a sizeable signal sample with the currently achievable luminosity. However, the process $H \rightarrow c \bar{c}$ is not only difficult to trigger, but also challenging to distinguish from large QCD multi-jet background. More sophisticated search strategies should be developed to reach the needed sensitivity for signal observation. First, the VBF channel has striking experimental signature where a central Higgs boson is accompanied by two light jets with a large rapidity gap. Second, the addition of the photon improves the trigger efficiency compared to what can be achieved using only multi-jet final states as well as suppresses the gluon-rich dominant multi-jet background. We therefore propose to search for the signal process

$$
p p \rightarrow q q H \gamma \quad \text { with } H \rightarrow c \bar{c} .
$$

Our signal process has distinctive features, which are characterized by two $c$-jets from the Higgs boson decay and an energetic photon in the central region, with two light jets separated by a large rapidity gap. The dominant background is QCD multi-jet production associated with 


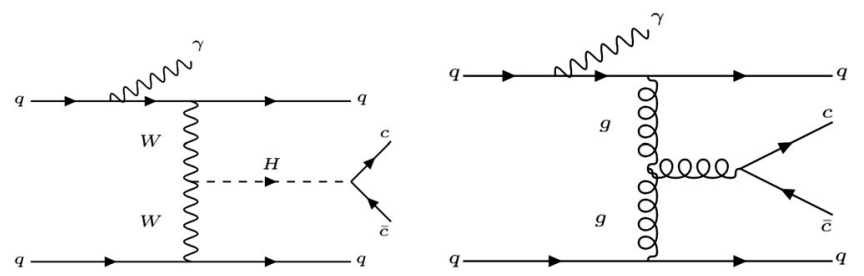

FIG. 1. Representative Feynman diagrams of the signal channel (left) and QCD multi-jet background (right).

a photon, where at least two jets are tagged (or mistagged) as $c$-jets. Other backgrounds include $Z \gamma+$ jets and VBF Higgs production $+\gamma$ with $H \rightarrow b \bar{b}$, where both $b$-jets are mistagged as $c$-jets. However, their contributions are expected to be much less significant than the QCD multi-jet background, thus not included in this analysis. The representative Feynman diagrams of the signal and leading background are shown in Fig. 1 for illustration.

Our analyses are designed to isolate the signal based on their kinematic features, described in detail in Sec. III. To estimate the achievable sensitivity to the signal at the hadronic collider environment, we use simulated events from Monte Carlo tools. With the very large hadronic production rate at the LHC, the trigger system is designed to record events of physical interest. For the relatively soft final states in our signal process, a dedicated trigger strategy is essential to record the signal events.

\section{A. Monte Carlo simulation}

Our targeted signal as seen in Eq. (1) is $H \rightarrow c \bar{c}$ plus an additional $\gamma$. Both signal and QCD multi-jet background are generated at LO with MG5@MCNLO v2.6.5 [56] at the $p p$ collider center of mass (c.m.) energy $\sqrt{s}=13 \mathrm{TeV}$ using the PDF4LHC15_nlo_mc PDFs [57]. The Higgs boson in the signal process is then decayed into $c \bar{c}$ by MadSpin [58]. The renormalization and factorization scales are set to be at the EW scale of the $W$-mass $\left(m_{W}\right)$. To enhance generation efficiency, both samples are generated with the following parton-level requirements, which are slightly looser than the thresholds used in analysis. We require two VBF jets inside the detector acceptance in pseudorapidity $(\eta)$, with transverse momenta

$$
p_{\mathrm{T}}^{j}>35 \mathrm{GeV}, \quad\left|\eta_{j}\right|<5,
$$

and an isolated photon in the central region with transverse momentum

$$
p_{\mathrm{T}}^{\gamma}>25 \mathrm{GeV}, \quad\left|\eta_{\gamma}\right|<3
$$

The parton shower and hadronization are simulated with PYTHIA 8 [59] and a fast detector simulation is implemented with DELPHES 3 using the default cards [60,61]. Jets are reconstructed using the anti- $k_{\mathrm{t}}$ algorithm [62] with a radius parameter
TABLE II. Cross sections of signal and background at different center-of-mass energies, with the basic acceptance cuts in Sec. II A.

\begin{tabular}{lcccc}
\hline \hline & $13 \mathrm{TeV}$ & $14 \mathrm{TeV}$ & $30 \mathrm{TeV}$ & $100 \mathrm{TeV}$ \\
\hline$\sigma_{\mathrm{VBF}+\gamma}(\mathrm{pb})$ & 0.024 & 0.027 & 0.099 & 0.43 \\
$\sigma_{p p \rightarrow 4 j+\gamma}(\mathrm{pb})$ & 830 & 940 & 3700 & 21000 \\
\hline \hline
\end{tabular}

$$
R=0.4 \text {. }
$$

After these basic acceptance cuts, the cross-sections of signal and leading background processes at different center-of-mass energies are listed in Table II using the same calculation set-up. We see that the signal rates are sizeable with the current and anticipated luminosities. However, the signal-to-background ratios are quite low, roughly at the order of $10^{-5}$, rendering the signal identification extremely challenging.

\section{B. Proposed trigger strategy}

The ATLAS [63] and CMS [64] experiments both contain a two-level trigger system. The first level, or level-1, trigger system is composed of custom electronics while the second level, or high-level trigger (HLT) runs software algorithms. The level-1 trigger systems of both experiments currently only utilize information from the calorimeter or muon subs-systems, and as a consequence, have shared items to trigger on electrons and photons [65]. Furthermore, due to the importance of selecting electrons with $p_{T} \approx m_{W} / 2$, approximately $25 \%$ of the total level-1 rate is devoted to electron/photon triggers. More information on the breakdown of rate and specific implementation can be found in the documents describing the ATLAS trigger menu [66-69].

After events have been selected from the level-1 trigger using a single EM object, these events can be used to seed a variety of triggers in the HLT, including requiring additional VBF jets or jets with a $b$-tag to reduce the rate. Relevant to our current considerations, the ATLAS analyses described in $[70,71]$ utilize a trigger with the following offline requirements:

(i) Photon $E_{\mathrm{T}}^{\gamma}>30 \mathrm{GeV}$;

(ii) At least four jets with $p_{\mathrm{T}}^{j}>40 \mathrm{GeV}$;

(iii) At least one pair of jets with $m_{j j}>700 \mathrm{GeV}$;

(iv) At least one $b$-tagged jet with $77 \%$ efficiency.

There are also VBF triggers described in $[69,72]$ with higher $m_{j j}$ threshold and jet $p_{T}$ threshold, which would have a lower acceptance for the genuine VBF events. To develop a trigger for $H \rightarrow c \bar{c}$ final states, it seems plausible that the trigger described above for $H \rightarrow b \bar{b}$ could be modified for charm final states, by either requiring a charm tag or by raising the $m_{j j}$ threshold. We leave the exact details and optimization to the experiments, and proceed 
by providing motivation for the use case of such a trigger.

\section{ANALYSES AND RESULTS}

\section{A. Cut-based analysis}

To obtain a physical intuition on the characteristics of our signal and background processes, we start with a simple cut-based analysis which utilize thresholds on different kinematic observables.
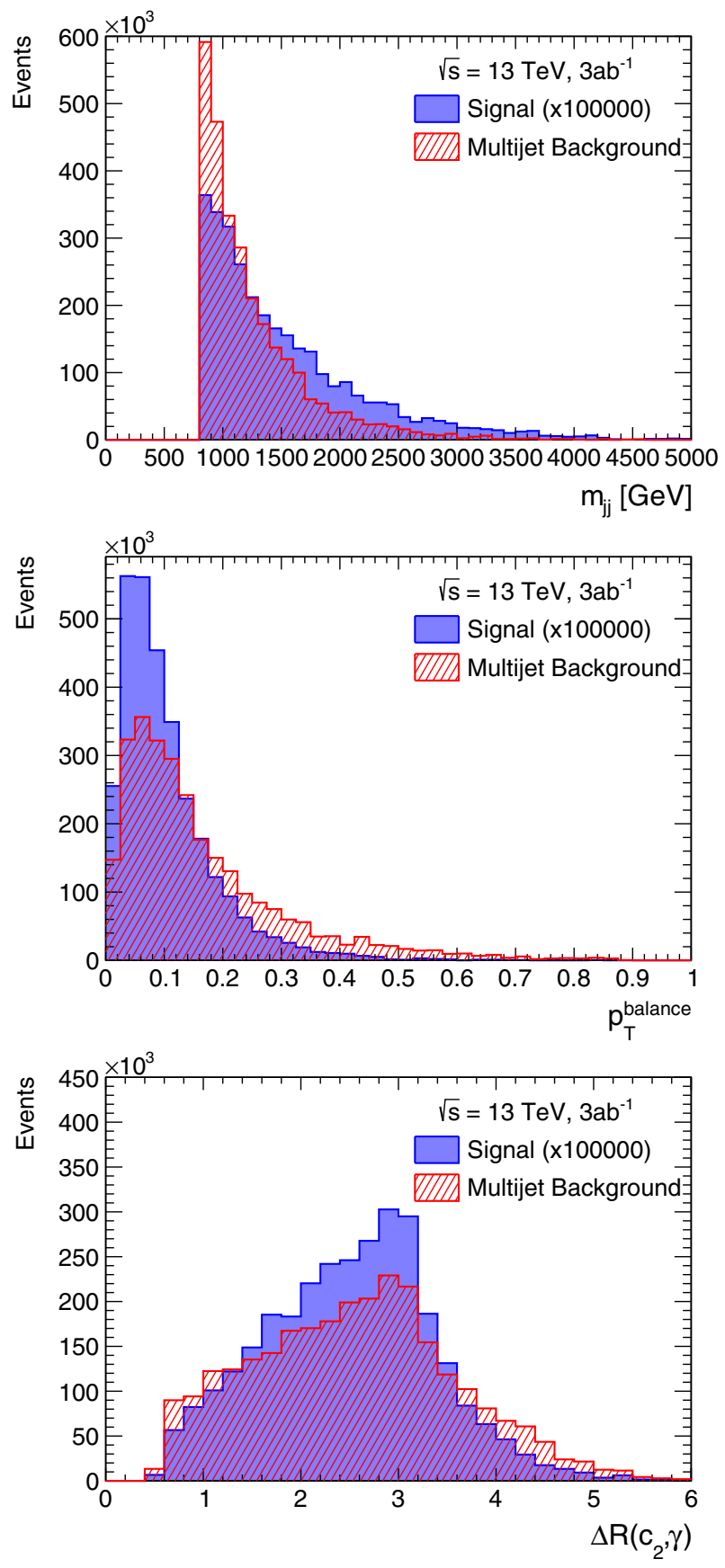

\section{Pre-selections}

The event pre-selections, inspired by [70,71], aim at capturing the basic features of a VBF signal. We require a photon in the central region with

$p_{\mathrm{T}}^{\gamma}>30 \mathrm{GeV}, \quad\left|\eta^{\gamma}\right|<1.37$ or $1.52<\left|\eta^{\gamma}\right|<2.37$.

Events are required to have 4 jets with

$$
p_{\mathrm{T}}^{j}>40 \mathrm{GeV} \text { and }\left|\eta^{j}\right|<4.4 \text {. }
$$
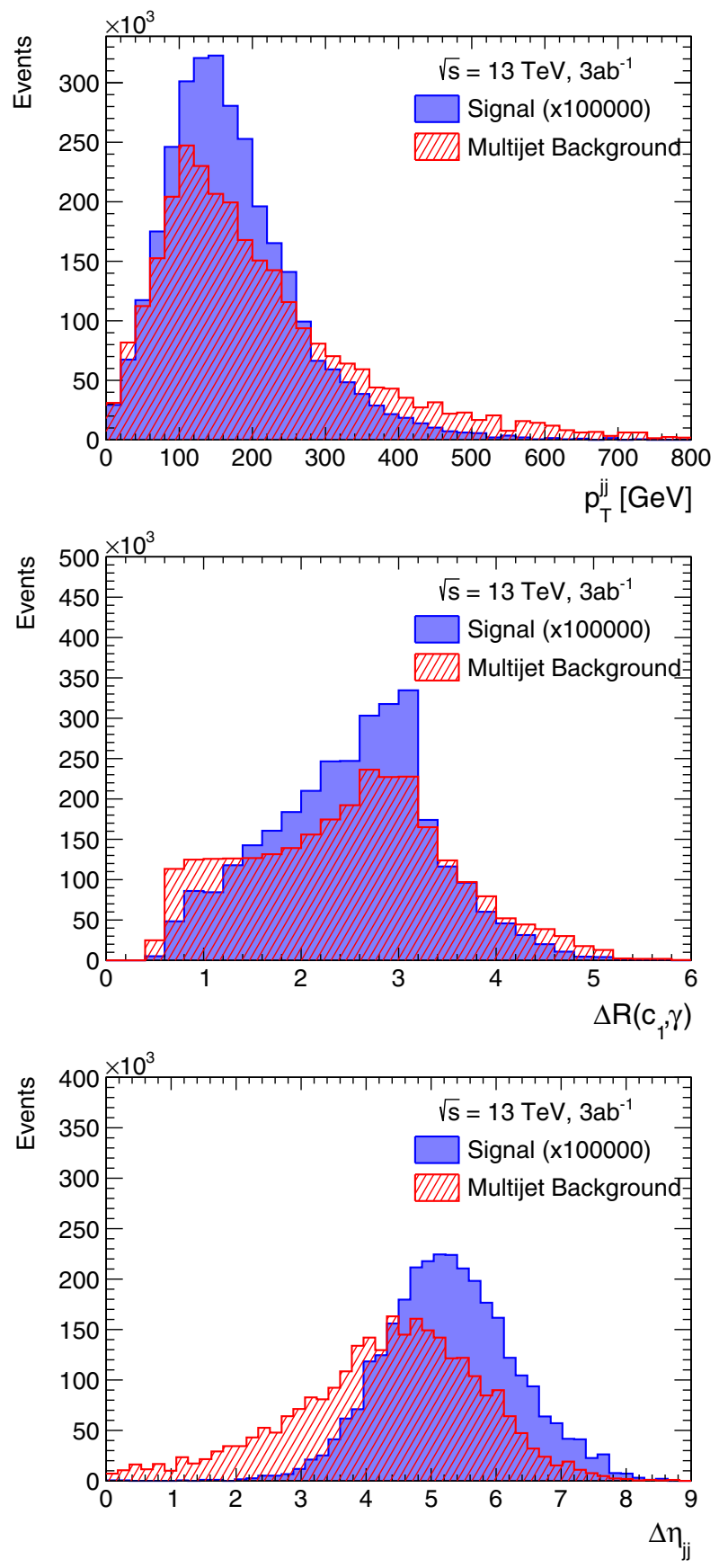

FIG. 2. Distributions of useful observables for signal (blue) and multi-jet background (red) after pre-selections. 
At least 2 jets in the central region $\left|\eta^{j}\right|<2.5$ are required to be $c$-tagged using fixed efficiency values depending on the truth flavor of the jet inspired by [17]. Charm jets are assumed to have a tagging efficiency of $41 \%$, while $b$-jets have a contamination probability of $25 \%$ and light jets a contamination probability of $5 \%$. The two highest- $p_{\mathrm{T}} c$ tagged jets are identified as signal jets from Higgs boson decay while the remaining jets are identified as the VBF jets. The VBF jet pair is required to have invariant mass of at least $800 \mathrm{GeV}$ so that the trigger requirement is fully efficient. In addition, the signal $c$-jet pair is required to have
$p_{\mathrm{T}}^{c c}>80 \mathrm{GeV}$ to remove potential bias in $m_{c c}$ distribution caused by the $p_{\mathrm{T}}^{j}$ thresholds in the trigger.

\section{Optimized analysis selections}

In order to exploit the full phase space of the kinematic features, we choose several additional kinematic observables which are useful to further distinguish signal and background. The distributions of these observables after the pre-selections are shown in Figs. 2 and 3. In addition to the pre-selections, we make some further judicious cuts based
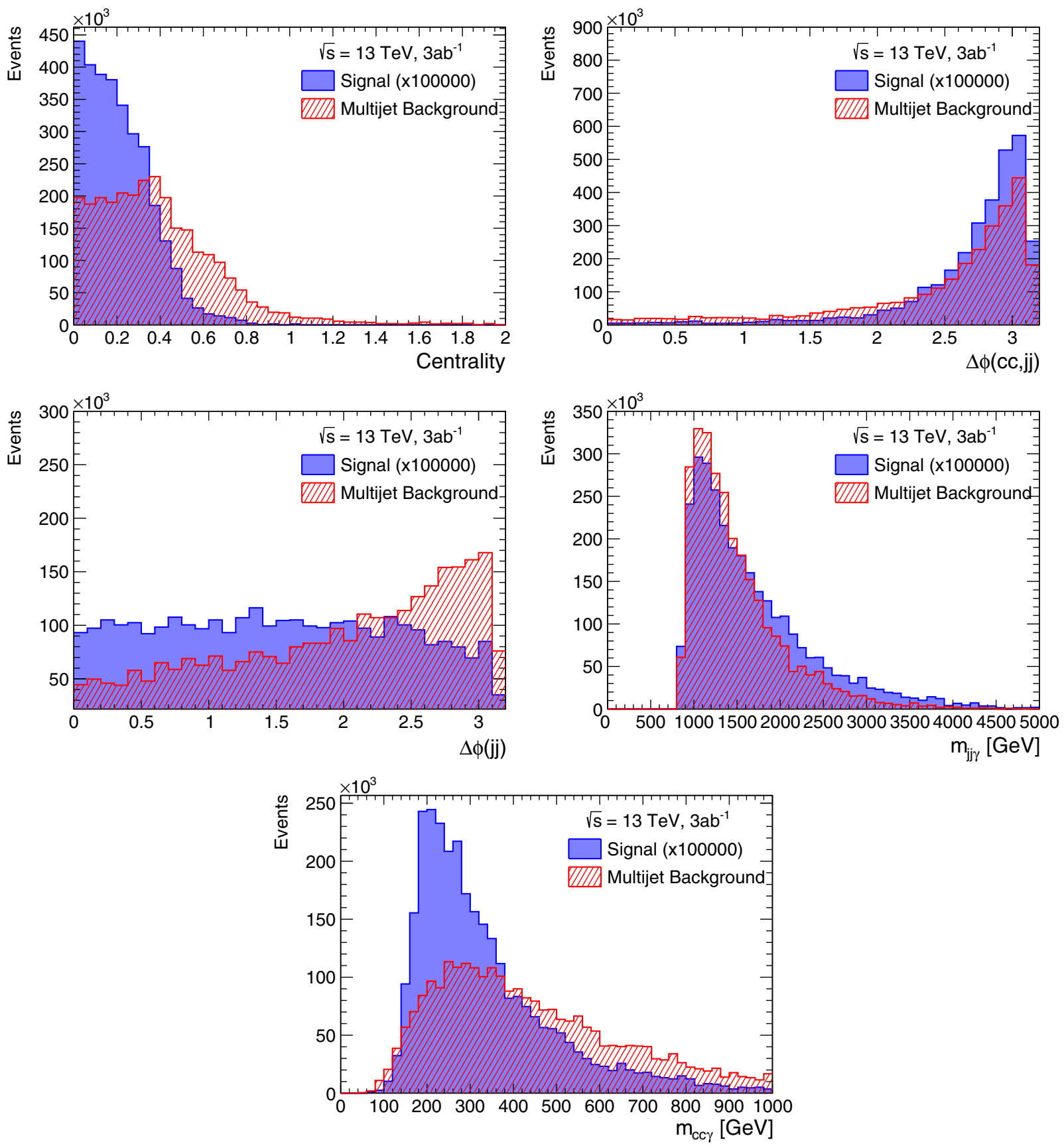

FIG. 3. Distributions of useful observables for signal (blue) and multi-jet background (red) after pre-selections. 
on the signal kinematics as follows. The invariant mass of the VBF jets (and photon) is large due to their back-to-back nature, so we require

$$
m_{j j}, \quad m_{j j \gamma}>1000 \mathrm{GeV} .
$$

The transverse momentum of the VBF jet pair is governed by the $W / Z$ exchange and thus relatively low. We limit their value to be

$$
p_{\mathrm{T}}^{j j}=\left|\vec{p}_{\mathrm{T}}^{j_{1}}+\vec{p}_{\mathrm{T}}^{j_{2}}\right|<300 \mathrm{GeV} .
$$

Since final states from electroweak processes tend to be more back-to-back than the QCD multi-jet background, we select events with the following ratio between the magnitudes of the vector and scalar sums of the jets and photon momenta

$$
p_{\mathrm{T}}^{\text {balance }}=\frac{\left|\vec{p}_{\mathrm{T}}^{j 1}+\vec{p}_{\mathrm{T}}^{j 2}+\vec{p}_{\mathrm{T}}^{c 1}+\vec{p}_{\mathrm{T}}^{c 2}+\vec{p}_{\mathrm{T}}^{\gamma}\right|}{p_{\mathrm{T}}^{j 1}+p_{\mathrm{T}}^{j 2}+p_{\mathrm{T}}^{c 1}+p_{\mathrm{T}}^{c 2}+p_{\mathrm{T}}^{\gamma}}<0.2
$$

Furthermore, because VBF signal features a large rapidity gap between the two forward-backward jets, events with large pseudo-rapidity separation between the two jets are selected for

$$
\Delta \eta_{j j}=\left|\eta_{j_{1}}-\eta_{j_{2}}\right|>4
$$

The reason that the multi-jet background also peaks at a relatively high value in Fig. 2 is due to the $m_{j j}$ requirement in the pre-selections.

As the photon is not radiated from $c$-jets from Higgs decay in our signal process, the angular separation between the signal $c$-jets and the photon tends to be larger in contrast to the QCD processes. Therefore, we require

$$
\Delta R\left(c_{1,2}, \gamma\right)>1.4
$$

where $c_{1,2}$ are leading and sub-leading $c$-jets. We also make use of the centrality of the photon relative to the VBF jets and require

$$
\text { centrality }=\left|\frac{y_{\gamma}-\frac{y_{j 1}+y_{j 2}}{2}}{y_{j 1}-y_{j 2}}\right|<0.35,
$$

where $y$ is the rapidity of the jet or photon [73]. Additionally, we utilize the azimuthal angular information on the transverse plane and require

$$
\Delta \phi(c c, j j)>2.3, \quad \Delta \phi(j j)<2.1
$$

It should be noted that the $\Delta \phi(j j)$, which is motivated by [74], has not been used to our knowledge before in the $H \rightarrow b \bar{b}$ searches. We then require the invariant mass of the $c$-jets and photon system

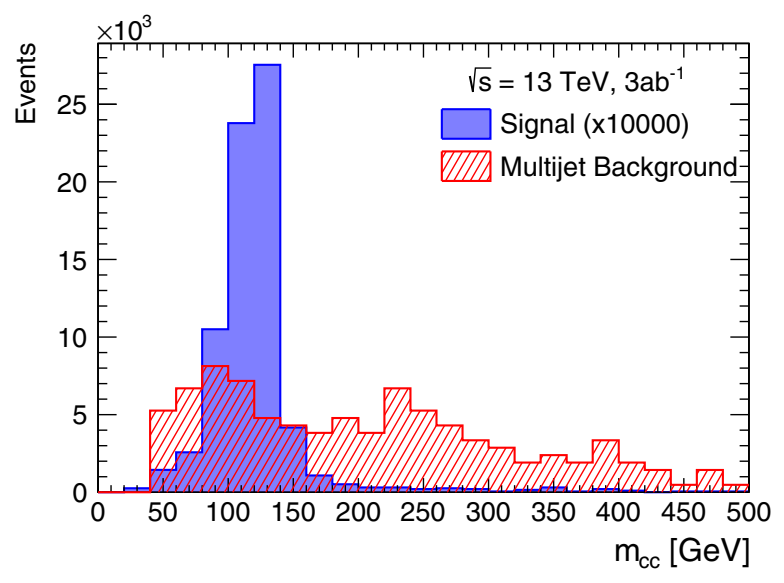

FIG. 4. Distribution of the invariant mass of the signal $c$-jet pair after cuts.

$$
m_{c c \gamma}<700 \mathrm{GeV}
$$

Since this observable is highly correlated with $m_{c c}$, we choose a relatively loose cut here.

The invariant mass of the signal $c$-jet pair $m_{c c}$ is used as the final discriminant. After the above selection requirements, the distribution of $m_{c c}$ is shown in Fig. 4. As expected, the Higgs signal peaks at $m_{H} \approx 125 \mathrm{GeV}$, while the multi-jet background has a more flat shape. Anticipating finite jet-mass resolution, we therefore require the $c$-jet pair to be in the Higgs mass window

$$
100 \mathrm{GeV}<m_{c c}<140 \mathrm{GeV}
$$

The expected numbers of events from signal and background of integrated luminosity $3 \mathrm{ab}^{-1}$ are shown in Table III. The truth flavor components of the $c$-tagged jet pairs in background are also shown in Table III. The major component is true $c$-jet pair as expected, but it should also be noted that the sub-leading components mainly involve light jets mistagged as $c$-jet. This suggests that improving the discrimination between $c$-jets and light jets can enhance the significance of such search.

TABLE III. Expected yields of signal and multi-jet background at the HL-LHC with $3 \mathrm{ab}^{-1}$ from a cut-based analysis.

\begin{tabular}{lcc}
\hline \hline & \multicolumn{2}{c}{ Cut-based } \\
\cline { 2 - 3 } & $\mathrm{S}$ & $\mathrm{B}$ \\
\hline Pre-selections & 31 & $2.8 \times 10^{6}$ \\
& & $(c c: 38 \%, c b: 5.6 \%, c j: 28 \%$, \\
& & $b b: 4.6 \%, b j: 5.7 \%, j j: 18 \%)$ \\
Optimized selections & 7.4 & $8.8 \times 10^{4}$ \\
Mass cut Eq. (15) & 5.1 & $1.2 \times 10^{4}$ \\
$S / \sqrt{B}$ & & 0.047 \\
\hline \hline
\end{tabular}



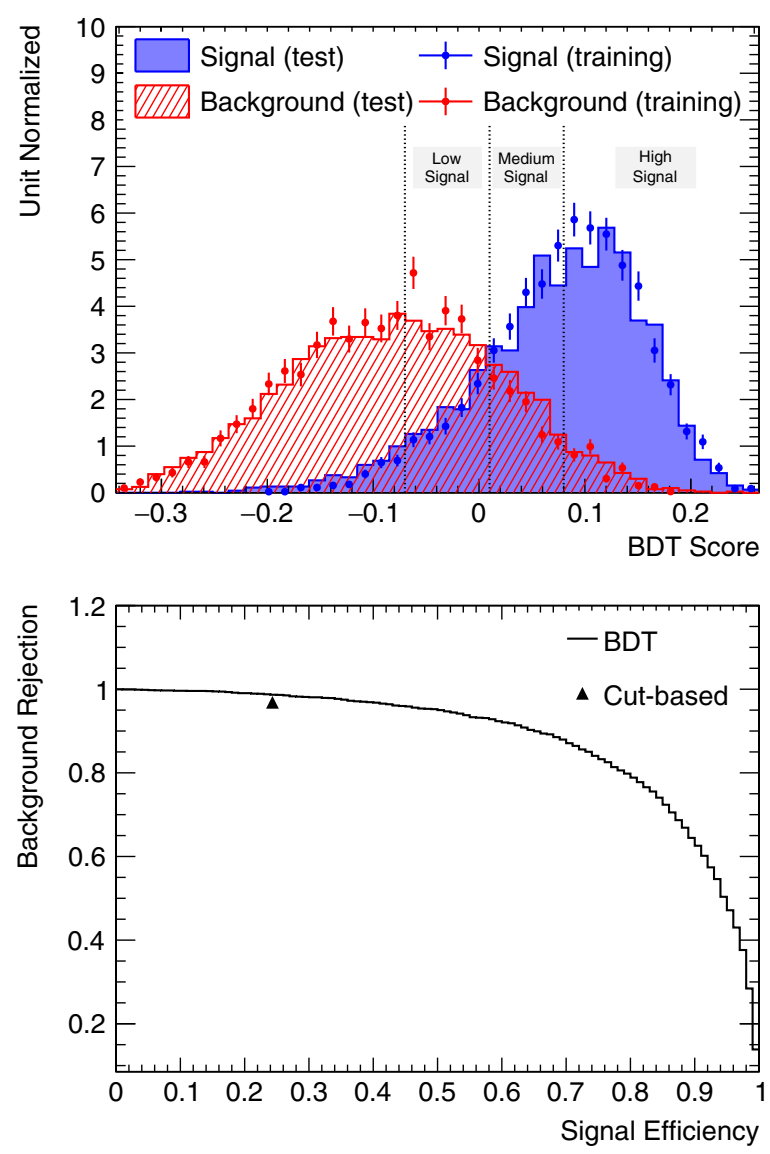

FIG. 5. The distribution of the BDT score with low, medium and high signal region defined as -0.07-0.01, 0.01-0.08 and $>0.08$ respectively (top) and receiver operating characteristic (ROC) curve of the BDT (bottom).

\section{B. Multivariate analysis}

Recent analyses of data in high energy physics have made extensive use of machine learning techniques, including the use of boosted decision trees (BDT) $[3,4,75]$. To improve the sensitivity reached by the simple cut-based studies in the last Sec. III A, a multivariate analysis is employed, starting with only the pre-selection cuts. A BDT is trained using the TMVA [76] package with the same set of observables shown in Figs. 2 and 3 as inputs.

The BDT is constructed by 850 trees, each with maximum depth of 3. A small depth is chosen because they are less susceptible to over-training but still perform very well with the aid of boosting algorithms. At each node of a tree, events are split into two subsets by cutting on an observable. The performance of the separation is assessed by Gini Index, defined by $p(1-p)$ where $p$ is the ratio of signal events to all events in that node. Therefore, a pure signal or background node corresponds to a zero Gini Index. The event sample is randomly split by half into training and test samples, where the former is used for BDT training and the latter is used for analysis and deriving limits. The distribution of the BDT score from signal and background along with a receiver operator characteristic (ROC) curve are shown in Fig. 5. The background rejection in the ROC curve on the right panel is defined as one minus the background survival probability after the selection cuts. The BDT performs as expected with a positive score as more "signal-like" and a negative score as more "background-like". We can see that the separation between signal and background is fairly well. The test sample distribution is superimposed with the training sample distribution, showing similar performance and thus indicates no occurrence of over-training.

To maximize the sensitivity, instead of a single cut, the BDT score is divided into three signal regions: low signal region with BDT score from -0.07 to 0.01 , medium signal region with BDT score from 0.01 to 0.08 , and high signal region with BDT score $>0.08$, as indicated in Table IV. The invariant mass distribution of the $c$-jet pair is not used in the BDT training but as a final discriminator and shown in Fig. 6. The invariant mass distribution $m_{c c}$ could be included in the BDT training to improve the separation between signal and background. However, as is commonly practiced by experiments, we reserved $m_{c c}$ as a most discriminative variable that can be used in a combined fit for signal plus background. A mass window of $100 \mathrm{GeV}-140 \mathrm{GeV}$ is again selected in the $m_{c c}$ distribution. The expected numbers of events from signal
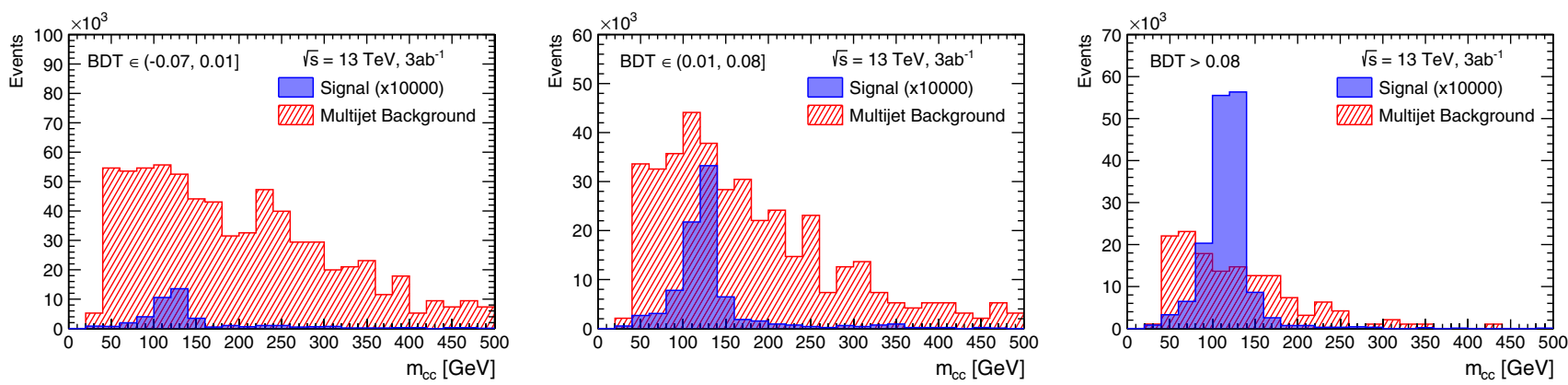

FIG. 6. Distribution of the invariant mass of the signal c-jet pair for events in different BDT score intervals, corresponding to the categories: low, medium and high signal. 
TABLE IV. Expected yields of signal and multi-jet background at the HL-LHC with $3 \mathrm{ab}^{-1}$ from a BDT analysis.

\begin{tabular}{|c|c|c|c|c|c|}
\hline & \multicolumn{2}{|c|}{ Low signal } & \multicolumn{2}{|c|}{ Medium signal } & High signal \\
\hline & $S$ & B & S & B & B \\
\hline BDT cut & 4.5 & $7.6 \times 10^{5}$ & 8.5 & $4.1 \times 10^{5}$ & $161.5 \times 10^{5}$ \\
\hline Mass cut Eq. (15) & 2.4 & $1.1 \times 10^{5}$ & 5.5 & $8.2 \times 10^{4}$ & $112.8 \times 10^{4}$ \\
\hline$S / \sqrt{B}$ & & 0.0073 & & 0.019 & 0.066 \\
\hline$S / \sqrt{B}$ combined & & & & 0.070 & \\
\hline
\end{tabular}

and background of integrated luminosity $3 \mathrm{ab}^{-1}$ are shown in Table IV for different BDT score intervals. With the BDT cut, we can reach a signal efficiency (background rejection in the interval of BDT score) of $15 \%$ (72\%), $28 \%$ $(85 \%)$ and $51 \%(95 \%)$ in the low, medium and high signal region respectively. In comparison, the signal efficiency (background rejection) of the optimized selection cuts in Sec. III A is $24 \%$ (97\%), shown together with the BDT ROC curve on the right panel in Fig. 5. For the same background rejection, BDT can achieve a signal efficiency of $40 \%$, outperforming the cut-based analysis. The overall significance is calculated by combining the significance in the three signal regions in quadrature, with the largest contribution coming from the high signal region. A relative change in significance of roughly $50 \%$ is seen, where the relative change is defined as $\left(\delta^{\mathrm{BDT}}-\delta^{\text {cut-based }}\right) / \delta^{\text {cut-based }}$, and $\delta=S / \sqrt{B}$.

Like the other extrapolations to the HL-LHC $[18,77]$, we have not considered the effects from the systematic errors. On the one hand, it is important to include the systematic effects to draw robust conclusions, especially given the rather small $S / B$ for the signal searches. On the other hand, the systematic effects due to the background measurement are largely unknown for the HL-LHC. We believe that when the large data sample becomes available, the systematic errors may be controlled to a desirable level of a few percent or lower.

\section{HL-LHC sensitivity to the charm-Yukawa coupling}

The expected $95 \% \mathrm{CL}_{s}$ upper limit on the signal strength $\mu$ in the absence of systematic uncertainties is approximated by $2 \sqrt{B} / S$. The BSM modification of the charm-Yukawa coupling is parametrized using the $\kappa$-scheme as

$$
y_{c}^{\mathrm{BSM}}=\kappa_{c} y_{c}^{\mathrm{SM}},
$$

then the number of signal events would approximately scale as

$$
N_{\text {sig }} \simeq \frac{\kappa_{c}^{2}}{\kappa_{H}^{2}} N_{\text {sig }}^{\mathrm{SM}}
$$

TABLE V. The expected $95 \% \mathrm{CL}_{s}$ upper limit on the signal strength and the charm-Yukawa coupling from this analysis using $3 \mathrm{ab}^{-1}$ of data at $13 \mathrm{TeV}$, respectively, in comparison with other searches as quoted.

\begin{tabular}{ccccccc}
\hline \hline & Cut-based & BDT & $Z H$ [18] & Fit [36] & $H c$ [34] & $H \rightarrow c \bar{c} \gamma[46]$ \\
\hline$\mu$ & 43 & 29 & 6.3 & $\ldots$ & $\ldots$ & 75 \\
$\kappa_{c}$ & $\cdots$ & 13 & 2.7 & 1.2 & $2.6-3.9$ & 8.8 \\
\hline \hline
\end{tabular}

where $\kappa_{H}$ denotes the BSM modification of the Higgs width. In principle, $\kappa_{H}$ depends on all BSM modifications of SM Higgs decay channels and any new channels. If we assume that $\kappa_{c}$ is the only non-SM modification, the upper limit on the signal strength can be translated into limit on the charm-Yukawa coupling:

$$
\mu=\frac{\kappa_{c}^{2}}{1+B r_{c c}^{\mathrm{SM}}\left(\kappa_{c}^{2}-1\right)},
$$

which are shown in Table V, in comparison with some of the existing literature.

\section{HE-LHC and $100 \mathrm{TeV}$ sensitivity to the charm-Yukawa coupling}

It is natural to ask to what extend the probe to the charmYukawa coupling can be improved at the future higher energy hadron colliders, such as the HE-LHC [78] and the FCC-hh [79]. The answer obviously depends on the detector performance of the charm-tagging, photon detection, and the QCD jet rejection, we nevertheless perform a crude estimate the sensitivity reach by assuming the same detector performance as the HL-LHC study. The sensitivity is estimated by extrapolating the HL-LHC performance as shown in the previous sections. We again calculate the signal cross section and the leading QCD background for $\sqrt{s}$ values of $30 \mathrm{TeV}$ and $100 \mathrm{TeV}$. By scaling the expected number of signal and background events for $\sqrt{s}=13 \mathrm{TeV}$ to higher energies, we extrapolate the sensitivities, assuming the same luminosity of $3 \mathrm{ab}^{-1}$ as shown in Table VI.

We have assumed that the cross section increase from $\sqrt{s}=13 \mathrm{TeV}$ to higher energy values does not change as a function of kinematic variables that are used as input to the BDT. Since both signal and background cross sections increase approximately linearly with center-of-mass energy, we see that the sensitivity scales roughly as the

TABLE VI. Expected sensitivity by scaling the collider c.m. energy $\sqrt{s}$.

\begin{tabular}{lccc}
\hline \hline$\sqrt{s}$ & $13 \mathrm{TeV}$ & $30 \mathrm{TeV}$ & $100 \mathrm{TeV}$ \\
\hline$S / \sqrt{B}\left(3 \mathrm{ab}^{-1}\right)$ & 0.07 & 0.14 & 0.25 \\
$\kappa_{c}$ reach & 13 & 5 & 3 \\
\hline \hline
\end{tabular}


square root of center-of-mass energy for the same integrated luminosity, reaching $S / \sqrt{B}$ of 0.25 and $\kappa_{c} \sim 3$.

\section{SUMMARY AND CONCLUSIONS}

Testing the charm-Yukawa coupling at the LHC is an important but very challenging task due to the overwhelmingly large QCD background. In this paper, we first reviewed the existing searches at the LHC and obtained the projection at the HL-LHC in probing the charmYukawa coupling, as summarized in Table I.

We proposed to study a new channel: the Higgs boson production via the VBF mechanism plus an additional hard photon in the hope to observe the challenging decay mode $H \rightarrow c \bar{c}$. The additional photon helps for the trigger to this hadronic decay process and to suppress gluon-rich QCD multi-jet background. The search that we proposed can utilize existing ATLAS and CMS triggers or offer new opportunities, for instance utilizing charm tagging in the HLT. We presented our specific proposal for the trigger design in Sec. II.

Based on the trigger considerations and the kinematic features of the signal, we first performed a cut-based analysis in Sec. III A, which yielded a sensitivity for signal strength $\mu$ of about 43 times the SM value at $95 \% \mathrm{CL}_{s}$ at the HL-LHC. A boosted decision tree described in Sec. III B enhanced the sensitivity by roughly $30 \%$ (using the same definition of relative change as used in Sec. III B), to about 29 times the SM value at $95 \% \mathrm{CL}_{s}$ at HL-LHC, corresponding to an upper limit of $y_{c}$ as 13 times the SM value.
Our obtained constraint on charm-Yukawa coupling, summarized in Table $\mathrm{V}$, is better than the $H \rightarrow J / \psi+\gamma$ channel [31]. Even though the limit obtained in our analyses is slightly weaker than the $Z H$ direct search by ATLAS [18], our channel will provide complementary information and a combination of different search channels can further improve the limit.

Global analyses of all the Higgs couplings [27,35-37] could result in a more sensitive probe than the direct search result $H \rightarrow c \bar{c}$, but admittedly depending on modeldependent assumptions, such as $\left|\kappa_{V}\right| \leq 1$ etc. Direct measurements of charm-Yukawa coupling are nevertheless indispensable.

Finally, we provided the first investigation of the VBF cross section with an associated photon at higher collider energies of $30 \mathrm{TeV}$ and $100 \mathrm{TeV}$. Assuming the same signal and background acceptance as well as the similar detector performance, some improvement of the sensitivity would be anticipated, as shown in Table V.

As we are entering the new phase of the LHC mission, it is important to push for the challenging measurements and to fully realize the potential for discovery at both the energy frontier and the precision frontier.

\section{ACKNOWLEDGMENTS}

This work was supported in part by the U.S. Department of Energy under Grant No. DE-FG02- 95ER40896, and in part by the PITT PACC.
[1] G. Aad et al. (ATLAS Collaboration), Observation of a new particle in the search for the standard model Higgs boson with the ATLAS detector at the LHC, Phys. Lett. B 716, 1 (2012).

[2] S. Chatrchyan et al. (CMS Collaboration), Observation of a new boson at a mass of $125 \mathrm{GeV}$ with the CMS experiment at the LHC, Phys. Lett. B 716, 30 (2012).

[3] A. M. Sirunyan et al. (CMS Collaboration), Observation of Higgs Boson Decay to Bottom Quarks, Phys. Rev. Lett. 121, 121801 (2018).

[4] G. Aad et al. (ATLAS Collaboration), Measurements of $W H$ and $Z H$ production in the $H \rightarrow b \bar{b}$ decay channel in $p p$ collisions at $13 \mathrm{TeV}$ with the ATLAS detector, Eur. Phys. J. C 81, 178 (2021).

[5] M. Aaboud et al. (ATLAS Collaboration), Observation of Higgs boson production in association with a top quark pair at the LHC with the ATLAS detector, Phys. Lett. B 784, 173 (2018).

[6] A. M. Sirunyan et al. (CMS Collaboration), Observation of țtH Production, Phys. Rev. Lett. 120, 231801 (2018).
[7] G. Aad et al. (ATLAS, CMS Collaborations), Measurements of the Higgs boson production and decay rates and constraints on its couplings from a combined ATLAS and CMS analysis of the LHC pp collision data at $\sqrt{s}=7$ and 8 TeV, J. High Energy Phys. 08 (2016) 045.

[8] M. Aaboud et al. (ATLAS Collaboration), Cross-section measurements of the Higgs boson decaying into a pair of $\tau$-leptons in proton-proton collisions at $\sqrt{s}=13 \mathrm{TeV}$ with the ATLAS detector, Phys. Rev. D 99, 072001 (2019).

[9] A. M. Sirunyan et al. (CMS Collaboration), Observation of the Higgs boson decay to a pair of $\tau$ leptons with the CMS detector, Phys. Lett. B 779, 283 (2018).

[10] D. Egana-Ugrinovic, S. Homiller, and P. R. Meade, Higgs bosons with large couplings to light quarks, Phys. Rev. D 100, 115041 (2019).

[11] S. Bar-Shalom and A. Soni, Universally enhanced lightquarks Yukawa couplings paradigm, Phys. Rev. D 98, 055001 (2018).

[12] T. Han and B. McElrath, $h \rightarrow \mu^{+} \mu^{-}$via gluon fusion at the LHC, Phys. Lett. B 528, 81 (2002). 
[13] T. Plehn and D. L. Rainwater, Higgs decays to muons in weak boson fusion, Phys. Lett. B 520, 108 (2001).

[14] Projections for measurements of Higgs boson cross sections, branching ratios, coupling parameters and mass with the ATLAS detector at the HL-LHC, Report No. ATLPHYS-PUB-2018-054, CERN, 2018.

[15] Sensitivity projections for Higgs boson properties measurements at the HL-LHC, Report No. CMS-PAS-FTR-18-011, CERN, 2018.

[16] A. Alves and F. F. Freitas, Towards recognizing the light facet of the Higgs boson, Mach. Learn. Sci. Tech. 1, 045025 (2020).

[17] M. Aaboud et al. (ATLAS), Search for the Decay of the Higgs Boson to Charm Quarks with the ATLAS Experiment, Phys. Rev. Lett. 120, 211802 (2018).

[18] Prospects for $H \rightarrow c \bar{c}$ using Charm tagging with the ATLAS experiment at the HL-LHC, Report No. ATL-PHYS-PUB2018-016, CERN, 2018.

[19] Direct constraint on the Higgs-charm coupling from a search for Higgs boson decays to charm quarks with the ATLAS detector, Report No. ATLAS-CONF-2021-021, CERN, 2021.

[20] A. M. Sirunyan et al. (CMS Collaboration), A search for the standard model Higgs boson decaying to charm quarks, J. High Energy Phys. 03 (2020) 131.

[21] X. Cid Vidal, C. Potterat, M. Santana Rangel, and L. Sestini, Search for $H^{0} \rightarrow b \bar{b}$ or $c \bar{c}$ in association with a $W$ or $Z$ boson in the forward region of $p p$ collisions, Report No. LHCb-CONF-2016-006, CERN, 2016.

[22] R. Aaij et al. (LHCb Collaboration), Physics case for an LHCb Upgrade II-Opportunities in flavour physics, and beyond, in the HL-LHC era, arXiv:1808.08865.

[23] G. Bodwin, F. Petriello, S. Stoynev, and M. Velasco, Higgs boson decays to quarkonia and the $h \bar{c} c$ coupling, Phys. Rev. D 88, 053003 (2013).

[24] G. T. Bodwin, H. S. Chung, J.-H. Ee, and J. Lee, New approach to the resummation of logarithms in Higgs-boson decays to a vector quarkonium plus a photon, Phys. Rev. D 95, 054018 (2017).

[25] G. T. Bodwin, H. S. Chung, J.-H. Ee, J. Lee, and F. Petriello, Relativistic corrections to Higgs boson decays to quarkonia, Phys. Rev. D 90, 113010 (2014).

[26] G. Perez, Y. Soreq, E. Stamou, and K. Tobioka, Prospects for measuring the Higgs boson coupling to light quarks, Phys. Rev. D 93, 013001 (2016).

[27] G. Perez, Y. Soreq, E. Stamou, and K. Tobioka, Constraining the charm Yukawa and Higgs-quark coupling universality, Phys. Rev. D 92, 033016 (2015).

[28] G. Aad et al. (ATLAS Collaboration), Search for Higgs and (Z) Boson Decays to $(J / \psi \gamma)$ and $(\Upsilon(n S) \gamma)$ with the ATLAS Detector, Phys. Rev. Lett. 114, 121801 (2015).

[29] M. Aaboud et al. (ATLAS Collaboration), Searches for exclusive Higgs and $Z$ boson decays into $J / \psi \gamma, \psi(2 S) \gamma$, and $\Upsilon(n S) \gamma$ at $\sqrt{s}=13 \mathrm{TeV}$ with the ATLAS detector, Phys. Lett. B 786, 134 (2018).

[30] A. M. Sirunyan et al. (CMS Collaboration), Search for rare decays of $\mathrm{Z}$ and Higgs bosons to $\mathrm{J} / \psi$ and a photon in proton-proton collisions at $\sqrt{s}=13 \mathrm{TeV}$, Eur. Phys. J. C 79, 94 (2019).
[31] Search for the standard model Higgs and Z boson decays to $J / \psi \gamma$ : HL-LHC projections, Report No. ATL-PHYS-PUB2015-043, CERN, 2015.

[32] M. König and M. Neubert, Exclusive radiative Higgs decays as probes of light-quark Yukawa couplings, J. High Energy Phys. 08 (2015) 012.

[33] M. Cepeda et al., Report from Working Group 2: Higgs physics at the HL-LHC and HE-LHC, CERN Yellow Rep. Monogr. 7, 221 (2019).

[34] I. Brivio, F. Goertz, and G. Isidori, Probing the Charm Quark Yukawa Coupling in Higgs + Charm Production, Phys. Rev. Lett. 115, 211801 (2015).

[35] N. M. Coyle, C. E. M. Wagner, and V. Wei, Bounding the charm Yukawa coupling, Phys. Rev. D 100, 073013 (2019).

[36] J. de Blas et al., Higgs boson studies at future particle colliders, J. High Energy Phys. 01 (2020) 139.

[37] L. M. Carpenter, T. Han, K. Hendricks, Z. Qian, and N. Zhou, Higgs boson decay to light jets at the LHC, Phys. Rev. D 95, 053003 (2017).

[38] Y. Soreq, H. X. Zhu, and J. Zupan, Light quark yukawa couplings from higgs kinematics, J. High Energy Phys. 12 (2016) 045.

[39] J. Cohen, S. Bar-Shalom, G. Eilam, and A. Soni, Lightquarks Yukawa couplings and new physics in exclusive high- $p_{T}$ Higgs boson + jet and Higgs boson $+\mathrm{b}$-jet events, Phys. Rev. D 97, 055014 (2018).

[40] F. Bishara, U. Haisch, P. F. Monni, and E. Re, Constraining Light-Quark Yukawa Couplings from Higgs Distributions, Phys. Rev. Lett. 118, 121801 (2017).

[41] A. M. Sirunyan et al. (CMS Collaboration), Measurement and interpretation of differential cross sections for Higgs boson production at $\sqrt{s}=13 \mathrm{TeV}$, Phys. Lett. B 792, 369 (2019).

[42] F. Yu, Phenomenology of enhanced light quark Yukawa couplings and the $W^{ \pm} h$ charge asymmetry, J. High Energy Phys. 02 (2017) 083.

[43] L. Alasfar, R. Corral Lopez, and R. Gröber, Probing Higgs couplings to light quarks via Higgs pair production, J. High Energy Phys. 11 (2019) 088.

[44] D. Egana-Ugrinovic, S. Homiller, and P. Meade, MultiHiggs production probes Higgs flavor, Phys. Rev. D 103, 115005 (2021).

[45] H. Khanpour, S. Khatibi, and M. Mohammadi Najafabadi, Probing Higgs boson couplings in $\mathrm{H}+\gamma$ production at the LHC, Phys. Lett. B 773, 462 (2017).

[46] T. Han, B. Nachman, and X. Wang, Charm-quark Yukawa coupling in $h \rightarrow c \bar{c} \gamma$ at LHC, Phys. Lett. B 793, 90 (2019).

[47] J. A. Aguilar-Saavedra, J. M. Cano, and J. M. No, More light on Higgs flavor at the LHC: Higgs couplings to light quarks through $h+\gamma$ production, Phys. Rev. D 103, 095023 (2021).

[48] B. Mele, Rescuing $H \rightarrow b \bar{b}$ in $\mathrm{VBF}$ at the $\mathrm{LHC}$ by requiring a central photon, in Proceedings of the 19th Conference on High Energy Physics (Springer, Milan, 2007).

[49] E. Gabrielli, F. Maltoni, B. Mele, M. Moretti, F. Piccinini, and R. Pittau, Higgs boson production in association with a photon in vector boson fusion at the LHC, Nucl. Phys. B781, 64 (2007). 
[50] F. Piccinini, $H \rightarrow b \bar{b}$ in VBF at the LHC with an extra central photon, Nuovo Cimento C 32N5-6, 323 (2009).

[51] D. Asner, M. Cunningham, S. Dejong, K. Randrianarivony, C. S. Rios, and M. Schram, Search for a light standard model Higgs boson produced in association with a photon in vector boson fusion, arXiv:1006.0669.

[52] K. Arnold, T. Figy, B. Jager, and D. Zeppenfeld, Higgs boson production in association with a photon via weak boson fusion, in Proceedings of the International Conference on the Structure and Interactions of the Photon and 19th International Workshop on Photon-Photon Collisions (2011).

[53] E. Gabrielli, B. Mele, F. Piccinini, and R. Pittau, Asking for an extra photon in Higgs production at the LHC and beyond, J. High Energy Phys. 07 (2016) 003.

[54] A. Biekötter, R. Gomez-Ambrosio, P. Gregg, F. Krauss, and M. Schönherr, Constraining SMEFT operators with associated $h \gamma$ production in weak boson fusion, Phys. Lett. B 814, 136079 (2021).

[55] D. de Florian et al. (LHC Higgs Cross Section Working Group), Handbook of LHC Higgs cross sections: 4. Deciphering the nature of the Higgs sector, arXiv:1610.07922.

[56] J. Alwall, R. Frederix, S. Frixione, V. Hirschi, F. Maltoni, O. Mattelaer, H. S. Shao, T. Stelzer, P. Torrielli, and M. Zaro, The automated computation of tree-level and next-toleading order differential cross sections, and their matching to parton shower simulations, J. High Energy Phys. 07 (2014) 079.

[57] J. Butterworth et al., PDF4LHC recommendations for LHC Run II, J. Phys. G 43, 023001 (2016).

[58] P. Artoisenet, R. Frederix, O. Mattelaer, and R. Rietkerk, Automatic spin-entangled decays of heavy resonances in Monte Carlo simulations, J. High Energy Phys. 03 (2013) 015 .

[59] T. Sjöstrand, S. Ask, J. R. Christiansen, R. Corke, N. Desai, P. Ilten, S. Mrenna, S. Prestel, C. O. Rasmussen, and P. Z. Skands, An introduction to PYTHIA 8.2, Comput. Phys. Commun. 191, 159 (2015).

[60] S. Ovyn, X. Rouby, and V. Lemaitre, DELPHES, a framework for fast simulation of a generic collider experiment, arXiv: 0903.2225 .

[61] J. de Favereau, C. Delaere, P. Demin, A. Giammanco, V. Lemaître, A. Mertens, and M. Selvaggi (DELPHES 3 Collaboration), DELPHES 3, A modular framework for fast simulation of a generic collider experiment, J. High Energy Phys. 02 (2014) 057.

[62] M. Cacciari, G. P. Salam, and G. Soyez, The anti- $k_{t}$ jet clustering algorithm, J. High Energy Phys. 04 (2008) 063.
[63] M. Aaboud et al. (ATLAS Collaboration), Performance of the ATLAS trigger system in 2015, Eur. Phys. J. C 77, 317 (2017).

[64] CMS Collaboration, The CMS trigger system, J. Instrum. 12, P01020 (2017).

[65] G. Aad et al. (ATLAS Collaboration), Performance of electron and photon triggers in ATLAS during LHC Run 2, Eur. Phys. J. C 80, 47 (2020).

[66] 2015 start-up trigger menu and initial performance assessment of the ATLAS trigger using Run-2 data, Report No. ATL-DAQ-PUB-2016-001, CERN, 2016.

[67] Trigger menu in 2016, Report No. ATL-DAQ-PUB-2017001, CERN, 2017.

[68] Trigger menu in 2017, Report No. ATL-DAQ-PUB-2018002, CERN, 2018.

[69] Trigger menu in 2018, Report No. ATL-DAQ-PUB-2019001, CERN, 2019.

[70] M. Aaboud et al. (ATLAS Collaboration), Search for Higgs bosons produced via vector-boson fusion and decaying into bottom quark pairs in $\sqrt{s}=13 \mathrm{TeV} p p$ collisions with the ATLAS detector, Phys. Rev. D 98, 052003 (2018).

[71] G. Aad et al. (ATLAS Collaboration), Search for Higgs boson production in association with a high-energy photon via vector-boson fusion with decay into bottom quark pairs at $\sqrt{s}=13 \mathrm{TeV}$ with the ATLAS detector, J. High Energy Phys. 03 (2021) 268.

[72] G. Aad et al. (ATLAS Collaboration), Performance of the ATLAS Level-1 topological trigger in Run 2, arXiv:2105 .01416 .

[73] For a massless object, the rapidity $y$ reduces to the pseudorapidity $\eta$.

[74] O. J. P. Eboli and D. Zeppenfeld, Observing an invisible Higgs boson, Phys. Lett. B 495, 147 (2000).

[75] V. M. Abazov et al. (D0 Collaboration), Evidence for Production of Single Top Quarks and First Direct Measurement of IVtbl, Phys. Rev. Lett. 98, 181802 (2007).

[76] A. Hoecker, J. Stelzer et al., TMVA-Toolkit for multivariate data analysis, arXiv:physics/0703039.

[77] Probing the $\mathcal{C P}$ nature of the Higgs boson coupling to $\tau$ leptons at HL-LHC, Report No. ATL-PHYS-PUB-2019008, CERN, 2019.

[78] A. Abada et al. (FCC Collaboration), HE-LHC: The highenergy large hadron collider: Future circular collider conceptual design report Volume 4, Eur. Phys. J. Special Topics 228, 1109 (2019).

[79] A. Abada et al. (FCC), FCC-hh: The hadron collider: Future circular collider conceptual design report Volume 3, Eur. Phys. J. Special Topics 228, 755 (2019). 